\title{
Australian Journal of

\section{Fertilization with potassium (K) in the soybean-corn succession affects behavior of plants and in the soil (oxisol)}

\author{
Edilson Cavalli*1, Anderson Lange ${ }^{2}$, Cassiano Cavalli ${ }^{1}$, Ivan Bedin ${ }^{3}$, Antonio Carlos Buchelt ${ }^{4}$, Estêvão Vicari \\ Mellis $^{5}$
}

${ }^{1}$ Postgraduate courses in tropical and subtropical agriculture, Agronomic institute -IAC, Campinas, São Paulo, Brazil ${ }^{2}$ Institute of Agrarian and Environmental Sciences, Federal university of Mato Grosso, Sinop, Mato Grosso, Brazil ${ }^{3}$ Rural Producer, Agronomist, Master in Agronomy, Sorriso, Mato Grosso, Brazil ${ }^{5}$ Postgraduate courses in agronomy, Paulista State University-UNESP, Jaboticabal, São Paulo, Brazil

${ }^{6}$ Agronomic institute -IAC, Campinas, São Paulo, Brazil

\section{*Corresponding author: edilso_c@hotmail.com}

\begin{abstract}
The potassium fertilizer recommendations are almost entirely made by the main culture in the field, without taking into account the predecessor and successor cultures. The nutrients in crop residues represent a reserve for subsequent crops. Therefore, knowledge of the absorption and cycling capacity of nutrient through the culture, and efficient fertilization should consider the entire system are important. This study aimed to evaluate the effect of potassium levels in soybean and corn, changes in nutrient stocks in the soil profile, absorption and accumulation in plant tissues and soybean, corn and Urochloa crop production during the two agricultural years. The study was performed in an oxisol in the cerrado-amazon ecotone, with potassium rates $(0,40,80,160$ and $320 \mathrm{~kg} \mathrm{ha}^{-1} \mathrm{~K}_{2} \mathrm{O}$ ). The rates applied in soybean (first crop) and three harvests of residual effect of the applied doses (maizesoybeans-maize) were evaluated. After harvesting the first crop, plots were subdivided (with or without the presence of Urochloa in corn spacing) in order to understand effect of Urochloa to increase the potassium cycling in the system. The grain yield and dry matter of Urochloa, extraction, and export of nutrients and their concentration in the leaf were evaluated. During the experiment, the chemical changes caused in the soil were also evaluated. In the first year, corn yield and the cumulative grain yield of the other crops were increasingly influenced by $\mathrm{K}_{2} \mathrm{O}$ rates. The rates provided increased $\mathrm{K}$ concentration and a decrease in $\mathrm{Ca}$ and $\mathrm{Mg}$ in plant materials. The $\mathrm{Ca}$ and $\mathrm{Mg}$ elements showed a decreased trend in the soil influenced by the applied rates of up to 130 days after fertilization. The $\mathrm{K}$ showed higher levels influenced by rates even after 619 days. We do not recommend the application of high doses of $\mathrm{K}$ due to the large losses caused by leaching.
\end{abstract}

Keywords: residual effect; export; extraction; leaching; Zea mays; Glycine max. Abbreviations: DAF- Days after fertilization

\section{Introduction}

The cultivation of soybeans and corn stands out globally for the planted areas and economic importance. In some regions of Brazil, climatic conditions favor the cultivation of the two crops in the same agricultural year, with soy being planted as the main crop and maize in sequence immediately after the soybean harvest. The researches on fertilization recommendation in the region is still incipient, in which data from other regions are being used. The recommendations should not be extrapolated from one region to another mainly for potassium fertilizer (Petter et al., 2012). The unknown response of cultures to the levels of nutrients in the soil and/or fertilizer applied urge a standardized application, year after year, regardless of soil conditions. The remaining nutrients in the waste of the previous crop cause unbalance and loss of productivity. The importance of potassium (K) for the development of crops is enormous, especially because plants extracted a large amount for their innumerable functions (Marschner, 2012). The response to fertilization is variable mainly due to the initial soil content and the production system (Scherer, 1998; Wendling et al., 2008; Takasu et al., 2014). In recent years, with the increase in productivity, especially in areas of second crop maize, it is possible to detect responses to potassium fertilizer in soil with low fertility and in rates of up to $120 \mathrm{~kg} \mathrm{ha}^{-1} \mathrm{~K}_{2} \mathrm{O}$ for corn (Coelho, 2006). Soybean typically has a lower response than corn, because of its less demanding. Thus, the $\mathrm{K}$ fertilization in areas with a suitable nutrient content in the soil, does not reflect gains in productivity and can face losses by leaching. In the state of Mato Grosso, these losses can be more expressive, because much of the cultivated soils present medium texture, having low CEC and especially high annual rainfall, concentrated especially in four months of the year, coinciding with the growth of the soybean and application of $\mathrm{K}$. 
This study aimed to evaluate the effect of potassium levels on soybean and corn, changes in nutrient stocks in the soil profile, leaching of potassium, the absorption and accumulation in plant tissues and soybean, corn and Urochloa crop production during the two agricultural years (2013-14 and 2014-15) under conditions of intense precipitation in the Cerrado

\section{Results and discussion}

\section{Effect on soil chemical properties}

The content of $\mathrm{Ca}^{2+}$ and $\mathrm{Mg}^{2+}$ nutrients in the first collection of soil and $\mathrm{K}^{+}$in the three samples were influenced by $\mathrm{K}$ rates applied, with no effect on $\mathrm{P}$ in any of the sampling (Fig 2, 3 and 4). The presence of Urochloa did not affect the nutrient content in the soil; thus, the results were not discussed.

The $\mathrm{Ca}^{2+}$ content was influenced down to layer of $0.15 \mathrm{~m}$, in $130 \mathrm{DAF}$ collection, and $\mathrm{Mg}^{2+}$ in the surface layer only (Fig 2). The application of potassium fertilizer resulted in decrease of these elements. The effect is much more pronounced for $\mathrm{Ca}^{2+}$, with a reduction of 20,39 and $32 \%$ content for layers 0 $0.05,0.05-0.10$ and $0.10-0.15 \mathrm{~m}$, respectively, compared to no fertilization that received $320 \mathrm{~kg} \mathrm{ha}^{-1} \mathrm{~K}_{2} \mathrm{O}$. In the same context, the $\mathrm{Mg}^{2+}$ was decreased by $15 \%$ in the $0-0.05 \mathrm{~m}$ layer.

Flora et al. (2007) explained the displacement behavior of $\mathrm{Ca}^{2+}$ and $\mathrm{Mg}^{2+}$ in soils by increasing the activity of $\mathrm{K}^{+}$. They noted that with the application of $\mathrm{K}$, there is 3.6 and 4.5 times increase in leaching of $\mathrm{Ca}^{2+}$ and $\mathrm{Mg}^{2+}$, respectively, comparing no potassium treatment and $400 \mathrm{~kg} \mathrm{ha}^{-1} \mathrm{~K}$ in the shape of $\mathrm{KCl}$ and $\mathrm{KNO}_{3}$.

The $\mathrm{K}$ presented linear trend at all times and layers evaluated (Fig 2, 3 and 4). The movement in the soil profile is widely reported in the literature. $\mathrm{K}$ is a nutrient that moves on the soil by diffusion. In high concentrations, it remains as solution on the soil; thus, it can be leached (Lange et al., 2008; Mendes et al., 2016). The accumulated precipitation from fertilization until the soil collection was 1,872, 2,127 and $4,265 \mathrm{~mm}$, for the first, second and third year, respectively, as the normal precipitation for the area. This precipitation rate facilitated vertical movement of fertilizers. Mendes et al. (2016) observed an increase in K leaching as increase in the applied water blade.

The highest $\mathrm{K}^{+}$values were observed in the 1 st and $2 \mathrm{nd}$ collections in the layer to $0.05 \mathrm{~m}$ depth at the rate of $320 \mathrm{~kg}$ $\mathrm{ha}^{-1} \mathrm{~K}_{2} \mathrm{O}$ with 181 and $169 \mathrm{mg} \mathrm{dm}^{-3}$, respectively. In the $3 \mathrm{rd}$ collection with the low stratification of layers and the marked decrease in $\mathrm{K}^{+}$content until $0.20 \mathrm{~m}$ deep, the highest content was observed in the layer 0.40 to $0.60 \mathrm{~m}$, $41.4 \mathrm{mg} \mathrm{dm}^{-3}$, also for the rate of $320 \mathrm{~kg} \mathrm{ha}_{-1} \mathrm{~K}_{2} \mathrm{O}$. These results demonstrate the significant leaching of $\mathrm{K}$, percolating through the surface layer to deeper layers, possibly, through the apparent depletion in the latter layer. It will be accumulated in the layers below $0.60 \mathrm{~m}$ deep.

We observed a decrease in the $\mathrm{K}^{+}$content in the soil in the course of time (Fig 5), with at the rates of 160 and $320 \mathrm{~kg}$ ha${ }^{1}$ of $\mathrm{K}_{2} \mathrm{O}$ in the $0-0.20 \mathrm{~m}$ layer having a top or near the initial content. This remained only in the first samplings but at the end of the evaluations the content ranged from 28.1 to 35.8 $\mathrm{mg} \mathrm{dm}^{-3}$, respectively for the zero rate and $320 \mathrm{~kg} \mathrm{ha}^{-1} \mathrm{~K}_{2} \mathrm{O}$. The $\mathrm{K}$ content remained above the initial content only in the layer of $0.40-0.60 \mathrm{~m}$ deep in the rate of $320 \mathrm{~kg} \mathrm{ha}^{-1}$ of $\mathrm{K}_{2} \mathrm{O}$ after 619 DAF. This reduction is associated with a great vertical $\mathrm{K}$ movement in the period, due to cumulative rainfall and application of 90,146 and $229 \mathrm{~kg} \mathrm{ha}^{-1} \mathrm{~K}$ in 1st, 2nd and 3 rd samplings, respectively. It requires $458 \mathrm{~kg} \mathrm{ha}^{-1}$ of $\mathrm{KCl}$ to replace the value of exports in the period.

After four crops, we observed accumulation of $222 \mathrm{~kg} \mathrm{ha}^{-1} \mathrm{~K}$ in treatment without initial addition of $\mathrm{K}$, and an export of $239 \mathrm{~kg} \mathrm{ha}^{-1} \mathrm{~K}$ to the treatment received $320 \mathrm{~kg} \mathrm{ha}^{-1} \mathrm{~K}_{2} \mathrm{O}$ (both of which received $80 \mathrm{~kg} \mathrm{ha}^{-1} \mathrm{~K}_{2} \mathrm{O}$ in corn crop 2013-14). The difference was only $17 \mathrm{~kg}$. So, the decrease in $\mathrm{K}^{+}$content in soil (Fig 5) is steeper for the higher rates, which is almost restricted to leaching of this element.

Scherer (1998) observed a gradual reduction of $\mathrm{K}$ reserves in the soils with initially $125 \mathrm{mg} \mathrm{dm}^{-3}$, which started to impair productivity after 4 years of cultivation. The same author confirmed that the treatments contents tend to equate during successive cultivations. This, conditioned largest export and most leaching in the treatments with the higher concentrations. This effect is shown in Fig 5, where in the 0$0.20 \mathrm{~m}$ layer the difference of $7.7 \mathrm{mg} \mathrm{dm}^{-3}$ between treatments was observed. It was very close to the levels in the final evaluation.

The most marked reduction in the content of $\mathrm{K}^{+}$is due to leaching during cultivation of the soybean, which was due to high precipitation during this period and the saturation with $\mathrm{K}$ at sites exchange in the soil. This is due to the large input via the mineral fertilizer and mineralization of corn. In corn growing season losses were smaller because maize had higher extraction capacity than soybean. The rain is diminished in March/April, when the corn is still absorbing K. Therefore, its roots reach deeper, recycling $\mathrm{K}$ where there is moisture. In conclusion, maize requires $\geq 80 \mathrm{~kg} \mathrm{ha}^{-1}$ of $\mathrm{K}$. The application should be recommended in the winter corn, as this requires $\mathrm{K}$ more than soybeans, and can store a large amount in their crop residues. The soil in this period shows less leaching, with better utilization of potassium fertilizer.

\section{Nutrient content in plant}

The application of $\mathrm{K}$ did not affect the potassium concentration in soybean leaves at 2013-14 harvest as well as the extraction and exportation by plant (Table 2). In 201314 , the corn production was increased linearly, when the rate applied to the leaf and extraction of nutrients. The accumulation of $\mathrm{K}$ in the residual straw was $207 \mathrm{~kg} \mathrm{ha}^{-1}$ in control, and $251 \mathrm{~kg} \mathrm{ha}^{-1}$ at a rate of $320 \mathrm{~kg} \mathrm{ha}^{-1} \mathrm{~K}_{2} \mathrm{O}$, which altered concentration of $\mathrm{K}$ in soybean leaf in sequence (2014-15). This did not alter the extraction and exportation. In 2014-15, the corn crop was only analyzed for the $\mathrm{K}$ concentrations in grain and its export by plant, in which there were no differences in concentration in the grain. In the 2 nd year of cultivation we observed lower concentration of $\mathrm{K}$ in compared to 1st year. This was due to the lower nutrient content in soil (Fig 5), and greater productivity in the harvest 2014-15 (Table 6) and possible dilution effect of absorbed $\mathrm{K}$.

The Urochloa was sown in consortium with corn. It responded to rates applied, reflecting an increased extraction. The soybean crop in 2013-14, showed a reduction in the concentration of $\mathrm{Ca}$ and $\mathrm{Mg}$ in the leaf influenced by $\mathrm{K}$ rates. We observed that only treatment without fertilization are distinguished from the others 
Table 1. Chemical and physical properties of oxisol.

\begin{tabular}{lrrrr}
\hline & \multicolumn{5}{c}{ Layers $(\mathrm{m})$} \\
\cline { 2 - 5 } & 0 to 0.10 & 0.10 to 0.20 & 0.20 to 0.40 & 0.40 to 0.60 \\
\hline Chemical & 5.44 & 5.43 & 5.23 & 5.24 \\
$\mathrm{pH}$ & 3.92 & 3.75 & 2.81 & 2.20 \\
$\mathrm{SOM}\left(\mathrm{g} \mathrm{dm}^{-3}\right)$ & 16.01 & 5.01 & 3.07 & 1.68 \\
$\mathrm{P}\left(\mathrm{mg} \mathrm{dm}^{-3}\right)$ & 95.60 & 84.00 & 66.20 & 40.00 \\
$\mathrm{~K}\left(\mathrm{mg} \mathrm{dm}^{-3}\right)$ & 4.00 & 2.18 & 1.19 & 0.70 \\
$\mathrm{Ca}\left(\mathrm{cmol}_{\mathrm{c}} \mathrm{dm}^{-3}\right)$ & 1.72 & 0.87 & 0.70 & 0.36 \\
$\mathrm{Mg}\left(\mathrm{cmol}_{\mathrm{c}} \mathrm{dm}^{-3}\right)$ & 7.34 & 6.46 & 6.10 & 3.90 \\
$\mathrm{H}+\mathrm{Al}\left(\mathrm{cmol}_{\mathrm{c}} \mathrm{dm}^{-3}\right)$ & 13.31 & 9.73 & 8.16 & 5.06 \\
$\mathrm{CEC}\left(\mathrm{cmol}_{\mathrm{c}} \mathrm{dm}^{-3}\right)$ & 44.86 & 33.59 & 25.23 & 22.98 \\
$\mathrm{~V}(\%)$ & & & & \\
\hline Physical & 530 & 553 & 586 & 607 \\
Clay $\left(\mathrm{g} \mathrm{kg}^{-1}\right)$ & 307 & 297 & 270 & 252 \\
Sand $\left(\mathrm{g} \mathrm{kg}^{-1}\right)$ & 163 & 150 & 144 & 141 \\
Silt $\left(\mathrm{g} \mathrm{kg}^{-1}\right)$ & 1.17 & 1.20 & - & - \\
Density $\left(\mathrm{g} \mathrm{cm}^{-3}\right)$ & 0.52 & 0.49 & - & - \\
Total Porosity $\left(\mathrm{m}^{3} \mathrm{~m}^{-3}\right)$ & 0.11 & 0.10 & - & - \\
Macroporosity $\left(\mathrm{m}^{3} \mathrm{~m}^{-3}\right)$ & 0.41 & 0.39 & - & - \\
Microporosity $\left(\mathrm{m}^{3} \mathrm{~m}^{-3}\right)$ & 76.12 & - & - & - \\
Hydraulic Conductivity $\left(\mathrm{mm} \mathrm{h}^{-1}\right)$ & & & & \\
\hline
\end{tabular}

$\mathrm{pH}$ (water); SOM, soil organic matter (oxi-reduction); $\mathrm{P}$ and $\mathrm{K}$ (Mehlich 1); $\mathrm{Ca}$ and $\mathrm{Mg}(\mathrm{KCl}) ; \mathrm{H}+\mathrm{Al}$ (calcium acetate); CEC, cation exchange capacity; $\mathrm{V}$, soil base saturation and particle size was measured using the pipette method.

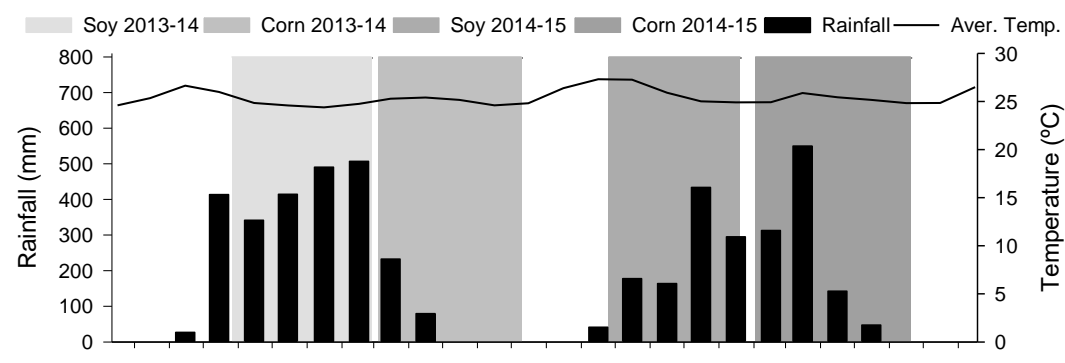

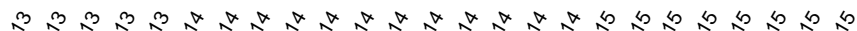

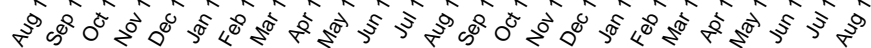

Fig 1. Rainfall and average temperature for Sorriso, from July 2013 to August 2015 (source: Mato Grosso Foundation).

Table 2. Leaf content, Extraction (Ext.) and Exporting (Exp.) of potassium for crops; soybeans 2013-14 and 2014-15, corn 2013-14 and 2014-15 and Urochloa (Uro.) and coefficients of variation (CV).

\begin{tabular}{|c|c|c|c|c|c|c|c|c|c|c|c|}
\hline & \multicolumn{3}{|c|}{ Soy 2013-14 } & \multicolumn{3}{|c|}{ Corn 2013-14 } & \multicolumn{3}{|c|}{ Soy $2014-15$} & \multirow{2}{*}{$\begin{array}{l}\text { Corn } \\
2014-15 \\
\text { Exp. } \\
\left(\mathrm{kg} \mathrm{ha}^{-1}\right)\end{array}$} & \multirow{2}{*}{$\begin{array}{l}\text { Uro. } \\
\text { Ext. } \\
\left(\mathrm{kg} \mathrm{ha}^{-1}\right)\end{array}$} \\
\hline & $\begin{array}{l}\text { Leaf } \\
\left(\mathrm{g} \mathrm{kg}^{-1}\right)\end{array}$ & $\begin{array}{l}\text { Ext. } \\
\left(\mathrm{kg} \mathrm{ha}^{-1}\right)\end{array}$ & Exp. & $\begin{array}{l}\text { Leaf } \\
\left(\mathrm{g} \mathrm{kg}^{-1}\right)\end{array}$ & $\begin{array}{l}\text { Ext. } \\
\left(\mathrm{kg} \mathrm{ha}^{-1}\right)\end{array}$ & Exp. & $\begin{array}{l}\text { Leaf } \\
\left(\mathrm{g} \mathrm{kg}^{-1}\right)\end{array}$ & $\begin{array}{l}\text { Ext. } \\
\left(\mathrm{kg} \mathrm{ha}^{-1}\right)\end{array}$ & Exp. & & \\
\hline \multicolumn{12}{|l|}{ Rates } \\
\hline 0 & 27.8 & 201.6 & 89.8 & 30.2 & 255.9 & 53.3 & 20.0 & 157.6 & 50.8 & 28.3 & 11.7 \\
\hline 40 & 26.1 & 205.5 & 91.1 & 30.2 & 276.4 & 55.5 & 20.3 & 170.7 & 54.0 & 29.2 & 12.0 \\
\hline 80 & 27.3 & 201.2 & 87.4 & 30.6 & 256.4 & 50.3 & 20.6 & 151.6 & 52.9 & 29.0 & 16.6 \\
\hline 160 & 27.9 & 198.6 & 89.8 & 30.6 & 304.8 & 65.5 & 22.2 & 160.9 & 54.9 & 31.2 & 16.1 \\
\hline 320 & 29.0 & 228.6 & 92.3 & 31.4 & 302.4 & 55.3 & 22.2 & 168.0 & 57.5 & 31.5 & 18.2 \\
\hline \multicolumn{12}{|c|}{ Consortium } \\
\hline With & - & - & - & 30.6 & 276.0 & 56.6 & 21.4 & 166.3 & 52.8 & 29.3 & - \\
\hline Without & - & - & - & 30.5 & 282.4 & 55.3 & 20.7 & 157.2 & 55.2 & 30.3 & - \\
\hline Average & 27.6 & 207.1 & 90.0 & 30.6 & 279.2 & 56.0 & 21.1 & 161.8 & 54.0 & 29.8 & 14.9 \\
\hline \multicolumn{12}{|c|}{ Statistical analysis } \\
\hline Rates & ns & ns & ns & $*(1)^{\#}$ & $*(2)$ & ns & $*(3)$ & $\mathrm{ns}$ & ns & $*(4)$ & $*(5)$ \\
\hline Cons. & - & - & - & ns & ns & ns & ns & ns & ns & ns & - \\
\hline $\mathrm{R} \times \mathrm{C}$ & - & - & - & ns & ns & ns & ns & ns & ns & ns & - \\
\hline $\mathrm{CV}_{1}(\%)$ & 7.32 & 18.01 & 4.25 & 2.16 & 12.71 & 20.70 & 6.22 & 17.54 & 11.07 & 6.57 & 20.59 \\
\hline $\mathrm{CV}_{2}(\%)$ & - & - & - & 1.81 & 11.06 & 21.40 & 7.01 & 16.02 & 10.24 & 10.81 & - \\
\hline
\end{tabular}




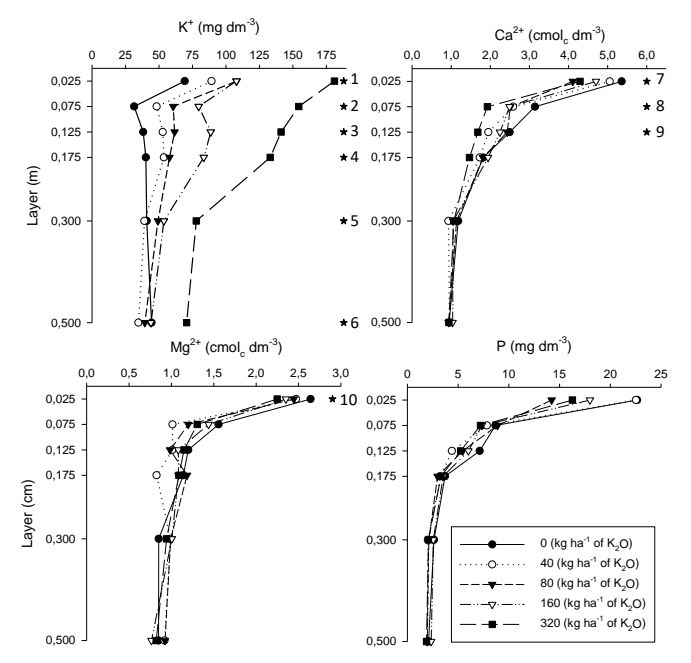

Fig 2: Influence of potassium rates on the nutrient content in different layers. Soil collected 130 days after fertilization. * and ** significant at the $5 \%$ and $1 \%$. Regression equations $1-\hat{y}=76.089+0.253 x, R^{2}=0.94 ; 2-\hat{y}=29.810+0.375 x, R^{2}=0.99 ; 3-\hat{y}=38.020+$ $0.320 x, R^{2}=1 ; 4-\hat{y}=38.890+0.289 x, R^{2}=1 ; 5-\hat{y}=37.605+0.121 x, R^{2}=0.97 ; 6-\hat{y}=34.960+0.097 x, R^{2}=0.77 ; 7-\hat{y}=5.011-0.002 x$, $R^{2}=0.40 ; 8-\hat{y}=2.904-0.003 x, R^{2}=0.84 ; 9-\hat{y}=2.398-0.002 x, R^{2}=0.54 ; 10-\hat{y}=2.559-0.001 x, R^{2}=0.85$.

Table 3. Leaf content, Extraction (Ext.) and exporting (Exp.) of calcium for crops; soybeans 2013-14 and 2014-15, corn 2013-14 and Urochloa (Uro.) and coefficients of variation (CV).

\begin{tabular}{|c|c|c|c|c|c|c|c|c|c|c|}
\hline & Soy 2013-14 & & & Corn 2013-14 & & & Soy $2014-15$ & & & Uro. \\
\hline & $\begin{array}{l}\text { Leaf } \\
\left(\mathrm{g} \mathrm{kg}^{-1}\right)\end{array}$ & $\begin{array}{l}\text { Ext. } \\
\left(\mathrm{kg} \mathrm{ha}^{-1}\right)\end{array}$ & Exp. & $\begin{array}{l}\text { Leaf } \\
\left(\mathrm{g} \mathrm{kg}^{-1}\right)\end{array}$ & $\begin{array}{l}\text { Ext. } \\
\left(\mathrm{kg} \mathrm{ha}^{-1}\right)\end{array}$ & Exp. & $\begin{array}{l}\text { Leaf } \\
\left(\mathrm{g} \mathrm{kg}^{-1}\right)\end{array}$ & $\begin{array}{l}\text { Ext. } \\
\left(\mathrm{kg} \mathrm{ha}^{-1}\right)\end{array}$ & Exp. & $\begin{array}{l}\text { Ext. } \\
\left(\mathrm{kg} \mathrm{ha}^{-1}\right)\end{array}$ \\
\hline \multicolumn{11}{|l|}{ Rates } \\
\hline 0 & $13.8 \mathrm{a}$ & 90.6 & 9.9 & 4.1 & 41.2 & 4.1 & 10.0 & 70.6 & 8.9 & 8.4 \\
\hline 40 & $9.4 \mathrm{~b}$ & 70.9 & 9.5 & 4.0 & 34.1 & 4.2 & 9.4 & 76.3 & 9.6 & 9.2 \\
\hline 80 & $10.1 \mathrm{~b}$ & 69.6 & 6.9 & 3.6 & 32.2 & 3.2 & 9.8 & 65.5 & 9.3 & 10.1 \\
\hline 160 & $11.3 \mathrm{~b}$ & 59.6 & 6.5 & 3.6 & 33.4 & 3.9 & 10.0 & 66.7 & 9.1 & 10.0 \\
\hline 320 & $10.3 \mathrm{~b}$ & 59.8 & 6.2 & 2.9 & 24.5 & 4.0 & 9.7 & 73.7 & 10.1 & 9.3 \\
\hline \multicolumn{11}{|c|}{ Consortium } \\
\hline With & - & - & - & 3.6 & 35.6 & 3.8 & $10.0 \mathrm{a}$ & 74.6 & 9.2 & - \\
\hline Without & - & - & - & 3.7 & 34.6 & 4.0 & $9.6 \mathrm{~b}$ & 66.5 & 9.6 & - \\
\hline Average & 11.0 & 70.1 & 7.8 & 3.7 & 35.1 & 3.9 & 9.8 & 70.6 & 9.4 & 9.54 \\
\hline \multicolumn{11}{|c|}{ Statistical analysis } \\
\hline Rates & $*(1)^{\#}$ & ns & $*(2)$ & $*(3)$ & ns & ns & ns & ns & ns & ns \\
\hline Cons. & - & - & - & ns & ns & ns & ${ }^{*}(4)$ & ns & ns & - \\
\hline $\mathrm{R} \times \mathrm{C}$ & - & - & - & ns & ns & ns & ns & ns & ns & - \\
\hline $\mathrm{CV}_{1}(\%)$ & 14.95 & 25.35 & 23.87 & 17.80 & 21.91 & 29.46 & 9.68 & 18.56 & 10.47 & 20.53 \\
\hline $\mathrm{CV}_{2}(\%)$ & - & - & - & 17.70 & 22.92 & 15.05 & 7.90 & 27.60 & 9.27 & - \\
\hline
\end{tabular}

ns not signficant, * significant at $5 \%$, equal letters in the column are not distinguished from each other $(p<0.05){ }^{*}$ regression equations $(1)-$ Scott Knott; $(2)-\hat{y}=10.07-$ $0.0364 x+0.000077 x^{2}, R^{2}=0.89 ;(3)-\hat{y}=4.11-0.00359 x, R^{2}=0.93 ;(4)$-Tukey.

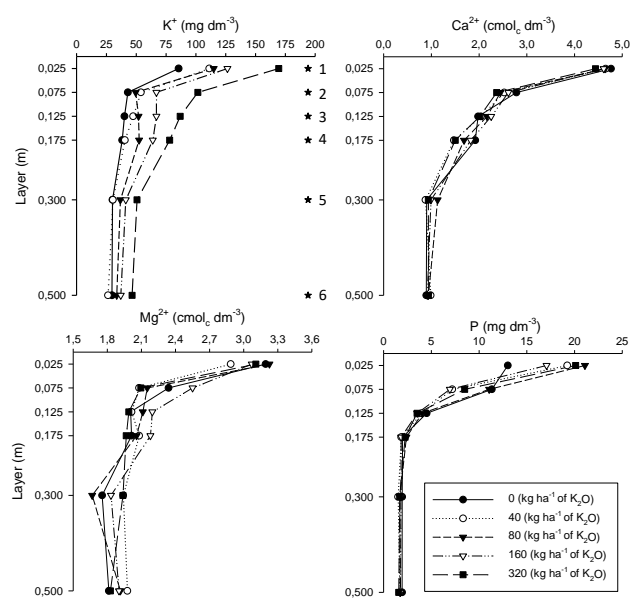

Fig 3. Influence of potassium rates on the nutrient content in different layers. Soil collected 334 days after fertilization. ${ }^{*}$ significant at $1 \%$. Regression equations $1-\hat{y}=90.735+0.278 x, R^{2}=0.96 ; 2-\hat{y}=41.335+0.180 x, R^{2}=0.96 ; 3-\hat{y}=41.078+0.146 x$, $R^{2}=0.99 ; 4-\hat{y}=39.145+0.129 x, R^{2}=0.96 ; 5-\hat{y}=29.820+0.067 x, R^{2}=0.97 ; 6-\hat{y}=27.513+0.059 x, R^{2}=0.92$. 
Table 4. Leaf content, Extraction (Ext.) and exporting (Exp.) of magnesium for crops; soybeans 2013-14 and 2014-15, corn 2013-14 and Urochloa (Uro.), significance value (p-value) and coefficients of variation (CV).

\begin{tabular}{|c|c|c|c|c|c|c|c|c|c|c|}
\hline & \multicolumn{3}{|l|}{ Soy 2013-14 } & \multicolumn{3}{|c|}{ Corn 2013-14 } & \multicolumn{3}{|c|}{ Soy 2014-15 } & \multirow{2}{*}{$\begin{array}{l}\text { Uro. } \\
\text { Ext. } \\
\left(\mathrm{kg} \mathrm{ha}^{-1}\right)\end{array}$} \\
\hline & $\begin{array}{l}\text { Leaf } \\
\left(\mathrm{g} \mathrm{kg}^{-1}\right)\end{array}$ & $\begin{array}{l}\text { Ext. } \\
\left(\mathrm{kg} \mathrm{ha}^{-1}\right)\end{array}$ & Exp. & $\begin{array}{l}\text { Leaf } \\
\left(\mathrm{g} \mathrm{kg}^{-1}\right)\end{array}$ & $\begin{array}{l}\text { Ext. } \\
\left(\mathrm{kg} \mathrm{ha}^{-1}\right)\end{array}$ & Exp. & $\begin{array}{l}\text { Leaf } \\
\left(\mathrm{g} \mathrm{kg}^{-1}\right)\end{array}$ & $\begin{array}{l}\text { Ext. } \\
\left(\mathrm{kg} \mathrm{ha}^{-1}\right)\end{array}$ & Exp. & \\
\hline \multicolumn{11}{|l|}{ Rates } \\
\hline 0 & $8.4 \mathrm{a}$ & 82.4 & 17.3 & 4.6 & 34.7 & 9.5 & 5.1 & 46.7 & 10.8 & 4.6 \\
\hline 40 & $6.0 \mathrm{~b}$ & 80.4 & 17.1 & 4.2 & 34.1 & 10.2 & 5.2 & 50.4 & 11.5 & 5.0 \\
\hline 80 & $6.8 \mathrm{~b}$ & 73.0 & 16.5 & 4.1 & 31.3 & 9.8 & 5.3 & 45.0 & 11.2 & 5.9 \\
\hline 160 & $7.4 \mathrm{~b}$ & 69.7 & 17.2 & 3.7 & 31.0 & 11.5 & 5.4 & 44.0 & 11.3 & 5.0 \\
\hline 320 & $6.8 \mathrm{~b}$ & 69.6 & 17.4 & 3.4 & 25.0 & 11.0 & 5.2 & 46.0 & 11.8 & 4.8 \\
\hline \multicolumn{11}{|c|}{ Consortium } \\
\hline With & - & - & - & 4.0 & 31.9 & 10.5 & 5.4 & 47.8 & 11.1 & - \\
\hline Without & - & - & - & 4.1 & 30.5 & 10.3 & 5.1 & 45.0 & 11.5 & - \\
\hline Average & 7.1 & 75.0 & 17.1 & 4.0 & 31.2 & 10.4 & 5.2 & 46.4 & 11.3 & 5.1 \\
\hline \multicolumn{11}{|c|}{ Statistical analysis } \\
\hline Rates & $*(1)$ & ns & ns & $*(2)^{\#}$ & ns & $*(3)$ & ns & ns & ns & ns \\
\hline Cons. & - & - & - & ns & ns & ns & ns & ns & ns & - \\
\hline $\mathrm{R} \times \mathrm{C}$ & - & - & - & ns & ns & ns & ns & ns & ns & - \\
\hline $\mathrm{CV}_{1}(\%)$ & 9.94 & 17.86 & 3.61 & 7.88 & 28.63 & 11.55 & 13.44 & 18.86 & 11.32 & 20.95 \\
\hline $\mathrm{CV}_{2}(\%)$ & - & - & - & 5.90 & 14.52 & 10.30 & 12.29 & 20.58 & 8.70 & - \\
\hline
\end{tabular}

(3) $-\hat{y}=9.36+0.01626 x-0.000034 x^{2}, R^{2}=0.77$.

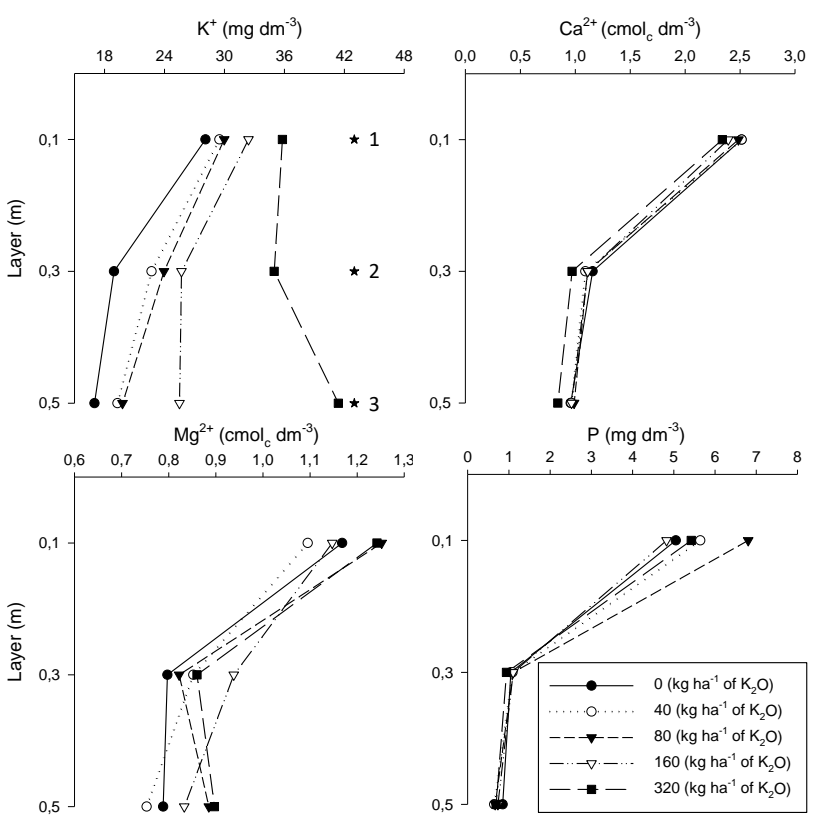

Fig 4. Influence of potassium rates on the nutrient content in different layers. Soil collected 619 days after fertilization. * and ** significant at the $5 \%$ and $1 \%$. Regression equations $1-\hat{y}=28.296+0.024 x, R^{2}=0.99 ; 2-\hat{y}=19.677+0.046 x, R^{2}=0.97 ; 3-\hat{y}=15.368+$ $0.077 x, R^{2}=0.97$.

Table 5. Leaf content, Extraction (Ext.) and exporting (Exp.) of nitrogen for crops; soybeans 2013-14 and 2014-15, corn 2013-14 and Urochloa (Uro.), significance value (p-value) and coefficients of variation (CV).

\begin{tabular}{|c|c|c|c|c|c|c|c|c|c|c|}
\hline & Soy 2013-14 & & & Corn 2013-14 & & & Soy 2014-15 & & & Uro. \\
\hline & $\begin{array}{l}\text { Leaf } \\
\left(\mathrm{g} \mathrm{kg}^{-1}\right)\end{array}$ & $\begin{array}{l}\text { Ext. } \\
\left(\mathrm{kg} \mathrm{ha}^{-1}\right)\end{array}$ & Exp. & $\begin{array}{l}\text { Leaf } \\
\left(\mathrm{g} \mathrm{kg}^{-1}\right) \\
\end{array}$ & $\begin{array}{l}\text { Ext. } \\
\left(\mathrm{kg} \mathrm{ha}^{-1}\right)\end{array}$ & Exp. & $\begin{array}{l}\text { Leaf } \\
\left(\mathrm{g} \mathrm{kg}^{-1}\right)\end{array}$ & $\begin{array}{l}\text { Ext. } \\
\left(\mathrm{kg} \mathrm{ha}^{-1}\right)\end{array}$ & Exp. & $\begin{array}{l}\text { Ext. } \\
\left(\mathrm{kg} \mathrm{ha}^{-1}\right) \\
\end{array}$ \\
\hline \multicolumn{11}{|l|}{ Rates } \\
\hline 0 & 39.8 & 391.1 & 194.9 & 23.4 & 133.6 & 92.8 & 39.3 & 274.7 & 157.1 & 14.3 \\
\hline 40 & 38.6 & 389.6 & 199.1 & 24.1 & 135.5 & 100.7 & 39.8 & 270.9 & 158.6 & 13.4 \\
\hline 80 & 38.5 & 390.6 & 195.9 & 24.6 & 134.5 & 98.9 & 39.2 & 252.7 & 152.6 & 17.4 \\
\hline 160 & 39.6 & 393.8 & 199.0 & 24.5 & 155.3 & 113.1 & 40.0 & 273.3 & 157.0 & 15.9 \\
\hline 320 & 40.1 & 411.1 & 196.7 & 24.1 & 154.1 & 110.3 & 40.6 & 276.0 & 158.8 & 14.0 \\
\hline \multicolumn{11}{|c|}{ Consortium } \\
\hline With & - & - & - & 24.2 & 144.1 & 103.3 & 39.5 & 275.5 & 154.3 & - \\
\hline Without & - & - & - & 24.0 & 141.1 & 103.1 & 40.0 & 263.5 & 159.4 & - \\
\hline Average & 39.3 & 395.3 & 197.1 & 24.1 & 142.6 & 103.2 & 39.8 & 269.5 & 156.8 & 15.0 \\
\hline \multicolumn{11}{|c|}{ Statistical analysis } \\
\hline Rates & ns & ns & ns & ns & ${ }^{*}(1)^{\#}$ & $*(2)$ & ns & ns & ns & ns \\
\hline Cons. & - & - & - & ns & $\mathrm{ns}$ & ns & ns & ns & ns & - \\
\hline $\mathrm{R} \times \mathrm{C}$ & - & - & - & $\mathrm{ns}$ & $\mathrm{ns}$ & ns & $\mathrm{ns}$ & ns & $\mathrm{ns}$ & - \\
\hline $\mathrm{CV}_{1}(\%)$ & 5.04 & 18.97 & 3.81 & 5.36 & 10.98 & 10.10 & 7.83 & 16.01 & 9.03 & 21.58 \\
\hline $\mathrm{CV}_{2}(\%)$ & - & - & - & 4.79 & 8.23 & 7.07 & 9.48 & 16.10 & 10.67 & - \\
\hline
\end{tabular}

ns not signficant, ${ }^{*}$ significant at $5 \%$, "regression equations $(1)-\hat{y}=133.73+0.074644 x, R^{2}=0.73 ;(2)-\hat{y}=92.44+0.16811 x-0.000347 x^{2}, R^{2}=0.88$ 

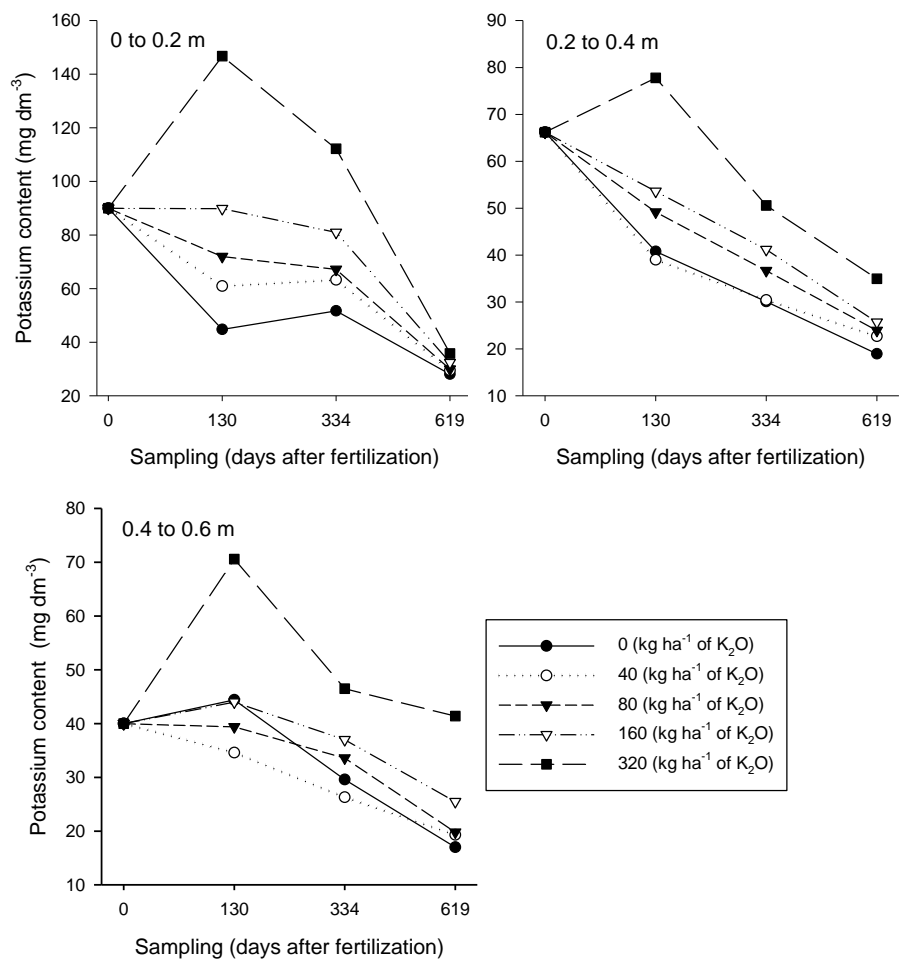

Fig 5. Behavior of potassium in the soil over time, influenced by the potassium rates employed.

Table 6. Grain yield for crops; soybean 2013-14 and 2014-15, corn 2013-14 and 2014-15, accumulated grains yield and dry matter production by Urochloa, significance values ( $p$-value) and coefficients of variation (CV).

\begin{tabular}{|c|c|c|c|c|c|c|}
\hline & $\begin{array}{l}\text { Soy } \\
2013-14\end{array}$ & $\begin{array}{l}\text { Soy } \\
2014-15\end{array}$ & $\begin{array}{l}\text { Corn } \\
2013-14\end{array}$ & $\begin{array}{l}\text { Corn } \\
2014-15\end{array}$ & Accumulated & Urochloa \\
\hline \multicolumn{7}{|l|}{ Rates } \\
\hline 0 & 3,608 & 3,055 & 7,214 & 9,653 & 23,530 & 1,082 \\
\hline 40 & 3,625 & 3,159 & 7,950 & 9,810 & 24,545 & 1,070 \\
\hline 80 & 3,517 & 3,008 & 8,118 & 9,795 & 24,438 & 1,281 \\
\hline 160 & 3,626 & 3,122 & 8,915 & 10,157 & 25,820 & 1,146 \\
\hline 320 & 3,642 & 3,246 & 8,777 & 10,099 & 25,763 & 1,224 \\
\hline \multicolumn{7}{|c|}{ Consortium } \\
\hline With & - & 3,062 & 8,208 & 10,047 & - & - \\
\hline Without & - & 3,174 & 8,182 & 9,759 & - & - \\
\hline Average & 3,604 & 3,118 & 8,195 & 9,903 & 24,816 & 1,161 \\
\hline \multicolumn{7}{|c|}{ Statistical analysis } \\
\hline Rates & ns & ns & $*(1)^{\#}$ & ns & $*(2)$ & ns \\
\hline Cons. & - & ns & ns & ns & - & - \\
\hline $\mathrm{R} \times \mathrm{C}$ & - & ns & ns & ns & - & - \\
\hline $\mathrm{CV}_{1}(\%)$ & 4.51 & 7.84 & 8.78 & 6.08 & 2.69 & 16.44 \\
\hline $\mathrm{CV}_{2}(\%)$ & - & 8.88 & 7.79 & 5.60 & - & - \\
\hline
\end{tabular}

and plants showed a concentration of $13.8 \mathrm{~g} \mathrm{~kg}^{-1}$ of $\mathrm{Ca}$ and $8.4 \mathrm{~g} \mathrm{~kg}^{-1} \mathrm{Mg}$ in the treatment without $\mathrm{K}$. In the other treatments, the concentrations were $10.3 \mathrm{~g} \mathrm{~kg}^{-1}$ of $\mathrm{Ca}$ and $6.8 \mathrm{~g} \mathrm{~kg}^{-1}$ of $\mathrm{Mg}$, with no adjustment equation (Tables 3 and 4). Normally, addition of $\mathrm{K}, \mathrm{Ca}$ or $\mathrm{Mg}$ results in a lower concentration of the remaining two cations, regardless of the crop grown (IPNI, 1998). For Ca we observed adjustment in the export total, whereas export was $9.9 \mathrm{~kg} \mathrm{ha}^{-1}$ in nofertilization treatment to $6.2 \mathrm{~kg} \mathrm{ha}^{-1}$ in the treatment receiving $320 \mathrm{~kg} \mathrm{ha}^{-1}$ of $\mathrm{K}_{2} \mathrm{O}$. As a note for soybean, the $\mathrm{K}$ application negatively affected the $\mathrm{Ca}$ and $\mathrm{Mg}$ absorption by plant, lowering the concentration of $\mathrm{Ca}$ and $\mathrm{Mg}$ in the leaf, and extraction of $\mathrm{Mg}$. However, it was still influenced by the increased productivity of corn in higher rates. We observed increase in export of $\mathrm{Mg}$ with increasing rates, with the maximum export rate of $254 \mathrm{~kg} \mathrm{ha}^{-1} \mathrm{~K}_{2} \mathrm{O}$. In the soybean with Urochloa consortium (2014-15), the concentration of $\mathrm{Ca}$ in the leaf was changed, wherein the previously cultivated Urochloa increased Ca concentration from $9.6 \mathrm{~g}$ $\mathrm{kg}^{-1}$ to 10.0. The Urochloa showed no response to applied rates.

The $\mathrm{K}$ application did not affect $\mathrm{N}$ in Urochloa and both 
soybean crops (Table 5). In corn crop (2013-14) the $N$ showed a similar behavior to the above-mentioned $\mathrm{Mg}$, wherein the $\mathrm{N}$ was influenced by the grains yield showed a linear increase in extraction and increase in export until the rate of $242 \mathrm{~kg} \mathrm{ha}^{-1} \mathrm{~K}_{2} \mathrm{O}$.

\section{Productivity}

Due to lack of rainfall after sowing Urochloa, the plant did not adequately developed and showed low mass productivity. This water deficit after reseeding $(30 \mathrm{~mm}$ in March, 79 in April $\mathrm{mm}$ and $0.0 \mathrm{~mm}$ in May), significantly reduced the accumulation and nutrient cycling, with no major agronomic effects for subsequent crops, with mass straw yield about $1.0 \mathrm{t} \mathrm{ha}^{-1}$ (Table 6).

The corn yields in the first crop (2013-14), and the total production of grain at the end of two growing was changed by applying $\mathrm{K}$, being the maximum rate response at $232 \mathrm{~kg}$ $\mathrm{ha}^{-1} \mathrm{~K}_{2} \mathrm{O}$ for corn and $252 \mathrm{~kg} \mathrm{ha}^{-1}$ of $\mathrm{K}_{2} \mathrm{O}$ for the accumulated yield. A similar response was reported by Petter et al. (2016), but the response was much more restricted, with the maximum yield obtained at a rate of $53 \mathrm{~kg} \mathrm{ha}^{-1} \mathrm{~K}_{2} \mathrm{O}$, where it was lower than this study.

The corn crop 2013-14 encountered conditions of low rainfall during development (only $227 \mathrm{~mm}$ ), when the last significant rain occurred 29 days after sowing, which reduced productivity to $8,190 \mathrm{~kg} \mathrm{ha}^{-1}$, compared to average historical yield of $8,520 \mathrm{~kg} \mathrm{ha}^{-1}$ in the last 5 years. Another factor was the late sowing, which did not occur in 2014-15 season, in which the average of the experiment was $9,902 \mathrm{~kg}$ ha $^{-1}$ as sowing in February 02, 2015 and accumulation of $1,053 \mathrm{~mm}$ of rainfall up to 98 days after sowing. In 2013-14 and 2014-15 crops, the average productivity of the MT state were 5,473 and $6,077 \mathrm{~kg} \mathrm{ha}^{-1}$ following the trend obtained in the experiment.

Comparison of productivity of both crops to regional studies shows promising, which is justified by the management model of the farm as reference in the region. Buchelt et al. (2013) obtained an average productivity of $6.328 \mathrm{~kg} \mathrm{ha}^{-1}$ and Lange et al. (2014) 6,394 kg ha-1. Brambilla et al. (2009) in 2007-08 season in an integration experiment, verified average productivity of $3.850 \mathrm{~kg} \mathrm{ha}^{-1}$ for corn under only 300 $\mathrm{mm}$ of accumulated rain and severe competition with Urochloa.

It is observed that excessive use of $\mathrm{K}$ or its application wrongly generates no real benefits to the producer, does not increase or maintains the $\mathrm{K}$ content in long-term soil, where a little productivity is affected, causing leaching to layers below $0.60 \mathrm{~m}$, compromising recycling by cover crops. The non-response of soybean to potassium fertilizer is in agreement with the literature (Scherer, 1998) imposing increasing $\mathrm{K}_{2} \mathrm{O}$ rates on soybeans and difference observed only 4 years after implantation for the rates. In the same region, Freddi et al. (2017) conducted an experiment with the principal component analysis in soil that was under succession of soybean-corn for 11 years. they observed that $\mathrm{K}$ content was one of the main factors influencing the soybean yield in soil that had $K$ deficiency, with the maximum observed content of $15.10 \mathrm{mg} \mathrm{dm}^{-3}$.

Response rates were noted only in corn but with low expression. The fact that the soybean is not influenced by rate or decrease of $\mathrm{K}$ content in the soil is connected to crop be sown on the residual straw of corn, which has a large pool of $\mathrm{K}$ and maintains an adequate supply of nutrients to soybean during the cycle. Cavalli et al. (2018), demonstrated that the corn straw releases $\mathrm{K}$ in quantity and in a synchronized manner with the needs of the soybean crop. The soil previously sampled before soybean planting which showed 90 and $52 \mathrm{mg} \mathrm{dm}^{-3} \mathrm{~K}$ stored in the residual straw at 0-0.20 m and 162 and $202 \mathrm{~kg} \mathrm{ha}^{-1}$ in 2013-14 and 2014-15 crops, respectively. The $2014-15$ crop showed the zero rate (lowest concentration of $\mathrm{K}$ accumulated in the straw). Thus the no response is consistent with the results presented by Scherer (1998) that explained when levels are between 60 and $80 \mathrm{mg} \mathrm{dm}^{-3}$, the soybean response to potassium fertilizer is very low or absent. The lowest content in crop 2014-15 is compensated by $\mathrm{K}$ present in the straw.

\section{Materials and methods}

\section{Site characterization}

The study was conducted in the municipality of Sorriso in the state of Mato Grosso ( $\mathrm{S}=12^{\circ} 31^{\prime} 06^{\prime}$ ", W $=55^{\circ} 40^{\prime} 22^{\prime \prime}$ and altitude of $365 \mathrm{~m}$ ) over the crop years 2013-14 and 2014-15. The climate is classified as Aw, warm tropical according to Köppen classification. It has two well-defined seasons, being the rainy summer and dry winter (Alvares et al., 2014). The climatic variations during the development of experiments are shown in Fig 1.

The experiment was carried out on an Oxisol. Prior to setting the experiment, on September 07, 2013 the soil was sampled and analyzed at layers; 0-0.10, 0.10-0.20, 0.20-0.40 and $0.40-0.60 \mathrm{~m}$ (recognition sampling) using a probe. Ten soil sub-samples were collected for subsequent homogenization and preparation of a composite sample, which was analyzed to have their chemical (EMBRAPA, 2017). Also, cylinders were withdrawn for physical analysis of soil, assessed through of average of ten samples (EMBRAPA, 2017; Table 1).

\section{Conduction of study and experimental design}

The experiment was implanted out on October 26, 2013, with sowing and fertilization of the soybeans. The treatments were at this time $\mathrm{K}_{2} \mathrm{O}$ rates $(0,40,80,160$ and $320 \mathrm{~kg} \mathrm{ha}^{-1}$ ) as $\mathrm{KCl}$, applied in surface in total area, with a randomized block design with five replications. The plots had a dimension of $6.6 \times 6 \mathrm{~m}$, sowing spaced $0.55 \mathrm{~m}$ between rows. At the time of sowing, the soil was covered with $14,176 \mathrm{~kg} \mathrm{ha}^{-1}$ of the residual dry mass of corn, containing $162 \mathrm{~kg} \mathrm{ha}^{-1} \mathrm{~K}$ in average.

The cultivar was the GB 874, fertilized with $20 \mathrm{~kg} \mathrm{ha}^{-1}$ of $\mathrm{N}$ in the form of urea $(44 \% \mathrm{~N})$ and $100 \mathrm{~kg} \mathrm{ha}^{-1}$ of $\mathrm{P}_{2} \mathrm{O}_{5}$ in the form of superphosphate $\left(18 \% \mathrm{P}_{2} \mathrm{O}_{5}, 16 \% \mathrm{Ca}\right.$ and $\left.8 \% \mathrm{~S}\right)$, before sowing over the whole area in surface. Harvest was held on February 27, 2014, and harvested $6.6 \mathrm{~m}^{2}$ per plot.

After harvesting soybeans, seeds of Urochloa ruzizienses and corn were split equally in half of subplots, causing the consortium with the subplots (measuring $3.3 \times 6 \mathrm{~m}$ ). To evaluate the residual effect of $K$ rates on soybeans, fertilization of corn was also performed in all the experiment, applied of $80 \mathrm{~kg} \mathrm{ha}^{-1}$ of $\mathrm{N}$ and $80 \mathrm{~kg} \mathrm{ha}^{-1}$ of $\mathrm{K}_{2} \mathrm{O}$ in the form of $400 \mathrm{~kg} \mathrm{ha}^{-1}$ commercial formulated (20-00-20). It applied 16 days after sowing (V2 stage), covering with 60 $\mathrm{kg}$ of $\mathrm{N}$ in the form of urea $(44 \% \mathrm{~N})$ and then 33 days after 
sowing (V5 stage). Sowing of braquiária was held before soybean harvest, on March 21, 2014, using 600 points of cultural value, but the lack of rain in the period did not allow a good establishment. The corn sowing was held on March 5, 2014, on the cultural remains of soybeans and the cultivar was the DKB 390 VT Pro 2 and the harvest was taken on 10 July 2014. After the harvest of corn in $6.6 \mathrm{~m}^{2}$, the plants were cut and accommodated in the area corresponding to a subplot, with the fractionated material into pieces of approximately $0.50 \mathrm{~m}$.

On September 25, 2014, soybean sowing on crop residues of corn was held to evaluate the residual effect of potassium fertilization and the use of $\mathrm{K}$ present in the corn straw and Urochloa. The cultivar was TMG 132, fertilized with $108 \mathrm{~kg}$ ha $^{-1}$ of $\mathrm{P}_{2} \mathrm{O}_{5}$ in the form of superphosphate $\left(18 \% \mathrm{P}_{2} \mathrm{O}_{5}, 16 \%\right.$ $\mathrm{Ca}$ and $8 \% \mathrm{~S}$ ), without the addition of $\mathrm{K}$ in pre-sowing. The harvesting took place on January 20, 2015, at $6.6 \mathrm{~m}^{2}$.

With the same purpose last year the sowing was carried out on February 2, 2015, on the cultural remains of the soybean. The cultivar was the DKB 245 RR, with the fertilization consisted of $100 \mathrm{~kg} \mathrm{ha}^{-1} \mathrm{~N}$ in urea form (44\% of $\mathrm{N}$ ) being applied half to half in the V2 and V4 stage, without the addition of $K$ at this time. The harvest was held on June 20, 2015 (6.6 $\mathrm{m}^{2}$ was harvested).

\section{Analysis of plant tissue}

In soybean we evaluated concentrations of the nutrients such as $\mathrm{K}, \mathrm{Ca}, \mathrm{Mg}$ and $\mathrm{N}$, in leaf, whole plant and the grain. The leaf was harvested following methodology proposed by Malavolta et al. (1997). The whole plant, except for the roots, was collected in a number of two plants per plot while staying within R7 stage, dried, weighed and the dry weight of these extrapolated to hectare. The grains were obtained at harvest and analyzed. With the above results we estimated extracted and exported nutrient value.

In the culture of the corn, the same nutrients as soybean (2013-14) were measured and the sampling was based on the recommendation by Malavolta et al. (1997). To determine the residual mass which is in the field, 10 whole plants were weighed (except grains and roots). With the above results we estimated extracted and exported nutrient value. In (2014-15) crop only the $\mathrm{K}$ concentration in the grain and export was analyzed.

Urochloa was collected manually on September 25, 2015, between corn lines, with five pitches from a template of 0.25 $\mathrm{m}^{2}$ on each parcel, cut close to the soil and oven dried. Its mass, concentration, and accumulation of $\mathrm{K}, \mathrm{Ca}, \mathrm{Mg}$ and $\mathrm{N}$ in tissue was quantified.

The analysis of $\mathrm{N}$ was conducted by the Kjeldahl method, and the other nutrients according to the methodology proposed by Malavolta et al. (1997).

\section{Analysis of soil nutrient content}

After seeding (October 26, 2013) and collection (March 05, 2014) of soybean, a soil sampling was carried out 130 days after fertilization (DAF) from the layers $0-0.05,0.05-0.10$, $0.10-0.15,0.15-0.20,0.20-0.40$ and $0.40-0.60 \mathrm{~m}$ in trench system. The trench was open on each portion $(1.0 \mathrm{~m}$ forward $\times 0.3 \mathrm{~m}$ wide $\times 0.6 \mathrm{~m}$ deep), held three subsamples per plot on the two side walls and an in the center of the trench, which was composed. The second sampling was performed after the corn harvest on September 25, 2014, 334 DAF, following the same as the previous collection procedure. The samples were taken in the subplots with and without Urochloa in point other than the first collection. The third collection was performed after harvest of the crop 2014-15 corn, on July 7, 2015, 619 DAF from the layers 0$0.20,0.20-0.40$ and $0.40-0.60 \mathrm{~m}$. It was done with the help of Dutch auger and comprised of 15 subsamples per plot.

The collected soil was air-dried, sieved into $2 \mathrm{~mm}$ sieve and thereafter was analyzed according to the methodology of EMBRAPA (2017).

\section{Statistical analysis}

The results were submitted to analysis of variance, when significant they were subjected to regression analysis, considering the effect of potassium rates to $5 \%$ level of probability. We used Scott Knott test for parameters that were significant by $\mathrm{F}$ test, and showed no adjustment in the regression analysis. For the factor; presence and absence of Urochloa, it was subjected to Tukey's test at 5\% probability when significant.

\section{Conclusion}

Potassium fertilization changed the $\mathrm{K}$ content in the soil with a linear increase in the rate and the residual effect observed up to 619 days after fertilization. With the addition of $K$ the displacement of $\mathrm{Ca}$ and $\mathrm{Mg}$ in soil exchange elapsed up to 130 days of fertilization. The modification of the nutrient content in soil is directly related to the concentration of nutrients in the tissue, which is more relevant in corn plants. Potassium fertilization reflected in corn crop productivity (2013-14) and cumulative production (soybean + corn) in both years. Soybean showed no responses to $\mathrm{K}$ fertilization, which occurs due to the large amount of $\mathrm{K}$ in corn stover, exceeding $160 \mathrm{~kg} \mathrm{ha}^{-1}$, which is available during the soybean crop.

\section{Acknowledgments}

We thank to the Foundation Mato Grosso in the person of Jose Antonio Costa for the availability of climate data, the Agrisus and FAPEMAT Foundation (process no. 477794/2011) for financial support for the implementation of the project, FAPEMAT by granting the first author scholarship.

\section{References}

Alvares CA, Stape JL, Sentelhas PC, Gonçalves JLM, Sparovek G (2014) Koppen's climate classification map for Brazil. Meteorol Z. 22: 711-728

Brady NC, Weil RR (2010) Elements of the nature and properties of soils. 3a ed. Boston: Pearson Educational Internation, $714 \mathrm{p}$.

Brambilla JA, Lange A, Buchelt AC, Massaroto JA (2009) Produtividade de milho safrinha no sistema de integração lavoura-pecuária, na região de Sorriso, Mato Grosso. Rev Bras M Sorgo, 8:263-274. 
Buchelt AC, Lange A, Bilibio F, Zanuzo MR, Cavalli E (2013) Milho safrinha integrado com Brachiaria ruziziensis e mecanismos de aplicação do fertilizante. Rev Ciênc Agroamb. 11:143-151.

Cavalli E, Lange A, Cavalli C, Behling M (2018) Decomposition and release of nutrients from crop residues on soybeanmaize cropping systems. Rev Brasil Cienc Agrar. 13:e5527.

Coelho AM (2006) Nutrição e Adubação do Milho. Sete Lagoas: Embrapa Milho e Sorgo. 10p. (Embrapa Milho e Sorgo. Circular Técnica, 78).

EMBRAPA (2017) Manual de Métodos de Análise de Solo Brasília, DF. 3a ed. Embrapa Informação Tecnológica, 573p.

Flora LPD, Ernani PR, Cassol PC (2007) Mobilidade de cátions e correção da acidez de um cambissolo em função da aplicação superficial de calcário combinado com sais de potássio. Rev Bras Cienc Solo. 31:1591-1598.

Freddi OS, Tavanti RFR, Soares MB, Almeida FT, Peres FSC (2017) Physical-chemical quality of a Latossol under direct seeding and soybean-corn succession in the cerradoamazonian ecotone. Rev Caatinga. 30: 991-1000.

IPNI (1998) Potassium for agriculture. Better Crops with Plant Food. 3:0-40

Lange A, Caione G, Schoninger EL, Silva RG (2014) Produtividade de milho safrinha em consórcio com capimmarandu em função de fontes e doses de nitrogênio em cobertura. Rev Bras M Sorgo, 13: 35-47.
Lange A, Cruz JC, Marques JJ (2008) Estoque de nutrientes no perfil do solo influênciados por doses de palha e nitrogênio no milho em semeadura direta. Rev Ciênc Agroamb. 6:29-38.

Malavolta E, Vitti GC, Oliveira AS (1997) Avaliação do estado nutricional das plantas. 2.ed. Piracicaba, Associação Brasileira para Pesquisa da Potássio e do Fosfato, 319p.

Marschner, $\mathrm{H}$. Mineral nutrition of higher plants. 3.ed London: Elsevier, 2012. 643p.

Mendes W C, Alves Júnior J, Cunha PCR, Silva AR, Evangelista AWP, Casaroli D (2016) Potassium leaching in different soils as a function of irrigation depths. R. Bras. Eng. Agríc. Ambiental, 20:972-977.

Petter FA, Andrade FR, Zuffo AM, Monteiro MMS, Pacheco LP, Almeida FA (2016) Doses e épocas de aplicação de potássio no desempenho agronômico do milho no cerrado piauiense. Com Sci. 7:372-382.

Petter FA, Silva JA, Pacheco LP, Almeida FA, Alcântara Neto F, Zuffo AM, Lima LB (2012) Desempenho agronômico da soja a doses e épocas de aplicação de potássio no cerrado piauiense. Rev Bras Cienc Agrar. 55:190-196.

Scherer EE (1998) Resposta da soja à adubação potássica em latossolo húmico distrófico num período de doze anos Rev Bras Cienc Solo, 22:49-55.

Wendling A, Eltz FLF, Cubilla MM, Amado TJC, Mielniczuk J (2008) Recomendação de adubação potássica para trigo, milho e soja sob sistema plantio direto no Paraguai. Rev Bras Cienc Solo. 32:1929-1939. 